\title{
A VALIDATED ALL-PRESSURE FLUID DROP MODEL AND LEWIS NUMBER EFFECTS FOR A BINARY MIXTURE'
}

\author{
K. Harstad* and J. Bellan**2 \\ Jet Propulsion Laboratory \\ California Institute of Technology \\ Pasadena. CA 91109
}

\begin{abstract}
The differences between subcritical liquid drop and supercritical fluid drop behavior are discussed. Under subcritical, evaporative high emission rate conditions, a film layer is present in the inner part of the drop surface which contributes to the unique determination of the boundary conditions; it is this film layer which contributes to the solution's convective-diffusive character. In contrast, under supercritical conditions the boundary conditions contain a degree of arbitrariness due to the absence of a surface, and the solution has then a purely diffusive character. Results from simulations of a free fluid drop under no-gravity conditions are compared to microgravity experimental data from suspended, large drop experiments at high, low and intermediary temperatures and in a range of pressures encompassing the sub- and supercritical regime. Despite the difference between the conditions of the simulations and the experiments (suspension vs. free floating), the time rate of variation of the drop diameter square is remarkably well predicted in the linear curve regime. The drop diameter is determined in the simulations from the location of the maximum density gradient, and agrees well with the data. It is also shown that the classical calculation of the Lewis number gives qualitatively erroneous results at supercritical conditions, but that an effective Lewis number previously defined gives qualitatively correct estimates of the length scales for heat and mass transfer at all pressures.
\end{abstract}

\section{Introduction}

Supercritical fluids are a common occurrence in propulsion systems, yet the fundamentals of their behavior is poorly understood. This is due to the unusual properties of supercritical fluids which combine the characteristics of both liquids and gases, making their behavior unintuitive. Furthermore, data obtained from (mostly optical) measurements can be misleading because regions of high density thus observed are frequently identified with liquids: a common misconception is that if

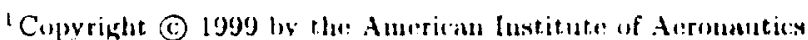
and Astrontubtics, Inc. All rights reserved

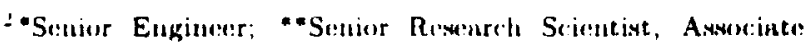
MAd Follow
}

in an experiment one can optically identify 'drops' and 'ligaments', the observed fluid must be in a liquid state. For example, Mayer et al. [1] and Krulle and Mayer [2] concluded that the $L O_{x}$ was in a liquid state. This inference is generally incorrect because in fact optical measurements detect any large change (i.e. gradients) in density. Thus, the density ratio may be well below $O\left(10^{3}\right)$ that characterizes its liquid/gas value, but the measurement will still identify a change in the index of refraction providing that the change is sudden (steep gradients). As shown by simulations of supercritical fluids [3], the density gradients may remain large during the supercritical binary fluids mixing, thus making them optically identifiable. Therefore, there is no inconsistency between the optical observation of high density regions and the fluids being in a supercritical state. A second misconception is that because a fluid has a liquid-like density, it is appropriate to model it as a liquid. However, such fluids may have liquid-like densities while their transport properties differ from those of a liquid. In fact, it was more recently observed and recognized that supercritical fluids behave very differently from liquids [4], [5] and [6] in that their breakup no longer resembles the well known atmospheric pressure atomization transforming a liquid sheet into approximately spherical drops.

Considering that the critical pressure of most fuel hydrocarbons used in Diesel and gas turbine engines is in the range of $1.5-3 \mathrm{MPa}$, and the fact that the maximum pressure attained in these engines is about 6 $\mathrm{MPa}$, it is clear that the fuel in the combustion chamber will experience both subcritical and supercritical conditions. Studies of drop behavior over a wide range of pressures were performed in the past (Yang et al. [8], Delplanque and Sirignano [9], Haldenwang et al. [10], and the review of Givler and Abraham (11]), however none of these studies identified the crucial differences between the subcritical and supercritical behavior. In fact, in two of these studies [8], $[10]$, it was found that the subcritical and supercritical behavior is similar as the drop diameter decreased according to the classical $d^{2}$-law [12] over a wide range of pressures and drop diameters. Moreover, despite the existence of microgravity data for model validation (see below), none of the existing models has undertaken a thorough comparison between numerical predictions and observations. 
The present stuly is devotied tos the exploration of differences in Huid-behavior characteristic's under subcritical and supercritical conditions, and to the model validation in both of these regimes. We choose to exercise the model for heptane Huid drops in uitrogen because of the availability of data for model validation.

\section{Model equations}

The configuration studied is that of a single spherical drop in a medium with specified far field conditions. These far field values are identified by the subscript ' $e$ ' and the location of the far field boundary, $R_{e}(t)$, is calculated in a Lagrangian way to be that of null mass flux.

The conservation equations are based upon Keizer's fluctuation theory [13] which has the distinct advantage of accounting for non-equilibrium processes. This formalism therefore leads to the most general fluid equations where the partial molar fluxes, $\vec{J}_{i}$, and the heat flux, $\vec{q}$, are related to thermodynamic quantities as follows:

$\vec{J}_{i}=L_{i q} \nabla \beta-\sum_{j}^{N} L_{i j} \nabla\left(\beta \mu_{j}\right), \quad \vec{q}=L_{q q} \nabla \beta-\sum_{j}^{N} L_{q j} \nabla\left(\beta \mu_{j}\right)$

where $\beta \equiv 1 /\left(R_{u} T\right), T$ is the temperature, $R_{u}$ is the universal gas constant, and $\mu_{j}$ are the chemical potentials. Here $L_{i j}$ are the Fick's diffusion elements, $L_{q q}$ is the Fourier thermal diffusion element, $L_{i q}$ are the Soret diffusion, $L_{q j}$ are the Dufour diffusion elements, and the Onsager relations state that $L_{i j}=L_{j i}$ and $L_{i q}=L_{q i}$. Additionally, conservation of fluxes and mass in the system imply that $\sum_{i}^{N} m_{i} \vec{J}_{i}=\overrightarrow{0}$ and $\sum_{i}^{N} L_{i j} m_{i}=0$ for $j \in[1, N]$ and $j=q$ where $m_{i}$ are the molar masses and $N$ is the total number of species.

Using the thermodynamic relationship

$$
d\left(\beta \mu_{j}\right)=\beta\left(v_{j} d p-h_{j} d \ln T\right)+\left(\sum_{1}^{N-1} \alpha_{D_{j i}} d X_{i}\right) / X_{j}
$$

where $v_{j}$ is the partial volume, $p$ is the pressure, $X_{i}$ is the molar fraction, $h_{j}$ is the molar enthalpy, and the mass diffusion factors are

$$
\alpha_{D i j} \equiv \beta X_{i} \partial \mu_{i} / \partial X_{j}=\partial X_{i} / \partial X_{j}+X_{i} \partial \ln \gamma_{i} / \partial X_{j}
$$

one can calculate $\vec{J}_{1}$ and $\vec{q}$ from 1 and 2 . The conservations equations were derived in detail in Harstad and Bellan [3] and results were obtained there for supercritical conditions. It was shown there that the transport matrix can be calculated as a function of the thermodynamic variables and of two transport matrices: one transport matrix is symmetric and is called the mass diffusivity matrix. How other transport mattrix is antisymmetric and is called the thermal diffusion factor matrix. The intricie'ies of the proper detinition of transport coeffic ient r that are consistent with experimentally measiured ones wiss indetressed in Harstad and Bellan [14. The emphasis is here on extending the calculations with the same model to the range of subcritical conditions. It turus out that one of the major differences between subcritical and supercritical regimes is in the boundary conditions at the drop boundary; it is this condition which is discussed below in detail, the two other boundary conditions (at the drop center and in the far field) being trivial.

\section{Boundary conditions}

The detailed boundary conditions at $r=R_{d}$ have been derived in [3]; here we describe only the new aspects that enabled the calculations to be extended to subcritical conditions. The jump conditions at the drop boundary are: mass balance; relationship between $R_{d}$ and the emission flux $F_{\text {ems }}$; heat balance; balance of species 1 flux; and the nonequilibrium evaporation law. Additional equations at $r=R_{d}$ are the momentum, and the equation of state for the mixture which is used twice (once on each side of the boundary). Thus there is a total of eight equations and nine unknowns: $u_{b}^{G}, X_{1 b}^{L}, X_{1 b}^{G}, \rho_{b}^{L}, \rho_{b}^{G}, R_{d}, T_{b}, p_{b}$ and $F_{e m s}$ where the subscript $b$ denotes the drop boundary, $\rho$ is the density, and superscripts $L$ and $G$ denote the inside and outside regions of the drop; variable $u_{b}^{L}$ is obtained by integrating the drop continuity equation starting at $r=0$. A ninth independent relationship exists only under subcritical conditions and is related to the existence of a surface, as discussed below.

The indeterminacy of the boundary conditions for a fluid drop under supercritical conditions has already been discussed by Harstad and Bellan [3]. This is physically understandable since there is no true surface, and thus there is an arbitrariness as to the choice of the boundary to follow. At least three choices are reasonable: One may follow the pure fluid boundary as was done by Harstad and Bellan [3]. Another possibility is to follow the initial boundary separating the two fluids, this being the choice in the present calculation. The third possibility is to follow the point of maximum density gradient; although this is not the present choice, the point of maximum density gradient is calculated here $d$ posteriori to indicate the location of the optically identified fluid drop. In contrast, under subcritical conditions the boundary to follow is the drop surface and the problem is fully determined.

There are other important consequences of the existence or lack of a surface at $r=R_{d}$. For example, under strong evaporative conditions a mass fraction 'film' layer exists inside the drop [15] and the thickness of this layer, $\delta_{Y} \ll \Delta r^{-}$where $\Delta r^{-}$is the distance from the surface to the first grid point inside the drop. A 


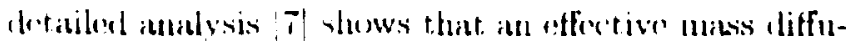
sivity $D_{\text {erf }}$ can be drined with the consecpuence that a tilu layer exist.y when $F_{\text {eng }} \gg \rho D_{\text {efs }} / \Delta r^{-}$. The value of $D_{\text {eff }}$ and that of an equivalent thermal conductivity $\lambda_{r}$ ff were calculated under the quasi-sterdy assumption in $[7 \mid$ by finding two linear combinations of $T$ and the mass fraction $Y_{1}$ for which the transport matrix can be approximately diagonalized. In diagonal form, the characteristic length scales for diffusional transport of these two new variables are apparent, and this allows the definition of $D_{e f f}$ and $\lambda_{e f f}$ [7]. Previous calculations [7] show that $\lambda_{\text {eff }} \geq \lambda$ and that $D_{\text {eff }} \leq D$. These definitions also allow the calculation of an effective Lewis number $L e_{\text {eff }} \equiv \lambda_{\text {eff }} /\left(n C_{p} D_{\text {eff }}\right)$ once the values of the dependent variables are known. The quasi-steady assumption does not remove the generality of the estimate since the essence of the estimate is that of a characteristic length.

One of the most important consequences of the mass fraction film layer existence is the direct relationship that exists between $Y\left(R_{d}-\varepsilon\right)$ and $Y\left(R_{d}+\varepsilon\right)$, where $\varepsilon \ll \max \left(\delta_{Y}, \delta_{T}\right)$; it is this relationship which provides the needed additional equation to fully determine the solution at the drop surface. This relationship can be formulated by considering the difference $\Delta Y_{1}^{L} \equiv Y_{1}^{L}\left(R_{d}-\varepsilon\right)-Y_{1}^{L}\left(R_{d}-\Delta r^{-}\right)$where $Y_{1}^{L}\left(R_{d}-\Delta r^{-}\right)$represents the computational grid center value at the first adjacent position to the film layer inside the drop such that $\Delta r^{-}>\delta_{Y}$. Similarly one may define $\Delta T^{L} \equiv T^{L}\left(R_{d}-\varepsilon\right)-T^{L}\left(R_{d}-\Delta r^{-}\right)$. The variable $\xi_{j} \equiv \exp \left(\varphi_{j}^{L}-\varphi_{j}^{G}\right)$, where $\varphi$ is the fugacity, quantifies the $Y_{j}$ jump across the drop surface and can be calculated from the state equation. For example, under strict equilibrium evaporation (i.e. $F_{e m s}=0$ ) conditions, $\xi_{j}=1$. For finite $F_{e m s}$ and for a binary mixture system, its ratio to a reference state $F_{\text {ref }}\left(\xi_{1}, \xi_{2}\right)$ can be defined by

$$
F_{\text {ems }}=\epsilon_{F} F_{r e f}
$$

where

$$
\begin{gathered}
F_{r e f} \equiv \mathcal{B}_{1}\left(\xi_{a v}-\xi_{1}\right)+\mathcal{B}_{2}\left(\xi_{2}-\xi_{a v}\right) \\
\mathcal{B}_{j} \equiv \alpha_{a j} m_{j} u_{T j} n^{C}, j=1,2 \\
X_{1}^{L}\left(R_{d}-\varepsilon\right)=\left(\xi_{2}-1\right) /\left(\xi_{2}-\xi_{1}\right)-\epsilon_{F} \\
X_{1}^{C}\left(R_{d}+\varepsilon\right)=\xi_{1}\left(\xi_{2}-1\right) /\left(\xi_{2}-\xi_{1}\right)-\xi_{a v} \epsilon_{F} \\
X_{2}^{L}\left(R_{d}-\varepsilon\right)=\left(1-\xi_{1}\right) /\left(\xi_{2}-\xi_{1}\right)+\epsilon_{F} \\
X_{2}^{C}\left(R_{d}+\varepsilon\right)=\xi_{2}\left(1-\xi_{1}\right) /\left(\xi_{2}-\xi_{1}\right)+\xi_{a v} \epsilon_{F}
\end{gathered}
$$

where $\alpha_{a j}$ is an accommodation coefficient, $u_{T j}$ is the mean molecular velocity crossing the plane in one direction, and $n$ is the number of moles per unit volume $\left[3 \mid\right.$ : consistently, $F_{\text {ref }}(1,1)=0$. A detailed analysis of the film layer yields then

$$
\begin{aligned}
\xi_{a v}= & {\left[\mathcal{B}_{2} \xi_{2} Y_{1}^{L}\left(R_{d}-\Delta r^{-}\right)+\mathcal{B}_{1} \xi_{1} Y_{2}^{L}\left(R_{d}-\right.\right.} \\
& \left.\left.\Delta r^{-}\right)\right] /\left[\mathcal{B}_{2} Y_{1}^{L}\left(R_{d}-\Delta r^{-}\right)+\mathcal{B}_{1} Y_{2}^{L}\left(R_{d}-\Delta r^{-}\right)\right]
\end{aligned}
$$

which provides the anditional relationship, that allows desure of the system of epluations at the drop boundary. Since cumbr supercritical conditions the concept of latent hout, and therefore of evaporation, is not applicable, the abour analysis does not hold. However. the film layer computational approach is still necessary if the Peclet number of the grid $P e_{g r 2 d} \geq O(1)$ in order to insure that all scales are resolved. Therefore, the formalism of the film layer is retained for computational purposes even under supercritical conditions, although the layer no longer exists physically. Essentially, the solution in the supercritical regime has a diffusive character, whereas in the subcritical regime it has a diffusive-convective character where the convective part is introduced by the film layer and the evaporation.

\section{Results}

The present simulations are performed for an n-heptane drop in nitrogen because it is the set of binary substances which is best documented experimentally. The equations of state have been calculated according to the procedure described in Harstad et al. [16], and the calculation of properties has been described in Harstad and Bellan [3]. The purpose of the present simulations is to validate the model; we also present here some results emphasizing the importance of the effective Lewis number concept.

The only data that can be used for comparisons is that obtained under evaporative rather than burning conditions, since in the last case the flame temperature that acts as the far field boundary is unknown. Furthermore, as shown below, it is only microgravity data that can be considered valid for these comparisons because normal gravity data has unavoidable convective effects that are not modeled here. However, Chauveau et al. (17) have shown that for the $10^{-2}-10^{-4}$ ratios of the microgravity to normal gravity obtainable both in parabolic flights and in drop tower experiments, buoyancy effects on the evaporation constant can still be identified. Additionally, since all high pressure microgravity drop evaporation experiments were performed with suspended drops, even these data are clearly not totally equivalent to our simulation results which are obtained for a free floating drop. The recent estimates of Morin [13] show that the effects of the suspending fiber increase with increasing pressure and temperature, and with decreasing drop size.

To our knowledge, microgravity obtained data with $C_{7} H_{16}$ drops evaporating in $N_{2}$ were reported only by Sato [19] and Nomura et al. [20]. In their experiments the $0.7-1 \mathrm{~mm}$ drops were suspended from a fiber of at least $100 \mu$ diameter. The $C_{7} H_{16}$ drop evaporation experiments of Chauveau et al. [21] were conducted only in normal gravity, whereas their reported microgravity experiments were of burning drops. Therefore, our comparison focuses on the data of [19] and [20], while also considering for reference (see Table 1) the more re- 


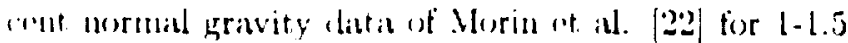
mun irops, instesul of that of Chauveau et al. [21].

The simulations were performed for nominal initial conditions matching the experimental data: $R_{d}^{0}=0.35$ mm except for the comprison with Sato's $[19 \mid$ data which was performed for $R_{d}^{0}=0.5 \mathrm{~mm}$, and $T_{d, b}^{()}=300$ $K$. The far tield conditions are located at $R_{e}^{0}=4 \mathrm{~mm}$ where $T_{e}$ and $p_{e}$ are specified consistent with those of the experiments and $Y_{e}^{0}=0$. The fluid drop is initially composed of pure heptane $\left(T_{c}=540.3 \mathrm{~K}, p_{c}=2.76\right.$ $\mathrm{MPa})$, while the surrounding is nitrogen $\left(T_{c}=126.2\right.$ $\mathrm{K}, p_{c}=3.39 \mathrm{MPa}$ ); in order to avoid an initial unphysical discontinuity, a minute amount of heptane exists initially in the drop surroundings, its distribution vanishing with increasing $r$. For the same reason, although the fluid drop temperature and drop-surroundings fluid composition are assumed initially uniform, a set of computational initial conditions (i.e. spatial profiles of the variables) are calculated for each simulation by satisfying the nominal initial conditions at the domain boundaries and the jump conditions at $R_{d}$.

In all of the discussions below, 'subcritical' and 'supercritical' qualifications will be used with respect to the heptane critical point, and not with respect to the critical point of the mixture which varies according to the local composition.

\section{Determination of thermal diffusion factors from high temperature data}

To proceed with the calculation, one must specify values for the thermal diffusion factors, $\alpha$, which can be defined either from the Irwing-Kirkwood (IK) or the Bearman-Kirkwood (BK) form of the heat flux [14] and $\alpha_{B K, i j}=\alpha_{I K, i j}-\alpha_{h, i j}$ where

$$
\alpha_{h, i j} \equiv\left(m_{i} m_{j} / m\right)\left(h_{i} / m_{i}-h_{j} / m_{j}\right) /\left(R_{u} T\right) .
$$

However, values of $\alpha_{B K}$ are not well known for most substances, except at atmospheric conditions where they can be calculated from kinetic theory. Since we are here interested in calculations at considerably larger pressures, the question arises as how to calculate $\alpha_{B K}$. For this purpose, the premise is that if it can be shown that $\alpha_{I K}$ is very small, in fact it can be considered negligible with respect to $\alpha_{B K}-\alpha_{I K}$, and then $\alpha_{B K} \simeq-\alpha_{h}$ which is only a function of thermodynamic quantities [14]. Since $\alpha_{h}$ is calculated from thermodynamics, this would provide an approximate value for $\alpha_{B K}$ for all $(p, T)$ conditions where $\alpha_{I K} / \alpha^{*}$ is very small having defined $\alpha^{*} \equiv \max _{\left(p, T, Y_{1}\right)}\left|\alpha_{h}\right|$. Heptane/nitrogen plots of $\alpha^{*}$ illustrated in $[14]$ show that $\alpha^{*} \sim 5$ at 0.1 and 0.5 $\mathrm{MPa}$ for $T \in[300 \mathrm{~K}, 800 \mathrm{~K}]$. Therefore, it is expected that $\alpha_{I K} / \alpha^{*} \ll 1$ is indeed correct in the range of $(p, T)$ of interest. The purpose of these high temperature data comparisons is to verify the consistency of the premise that $\alpha_{I K} / \alpha^{*}$ is very small. A similar premise for $\alpha_{B K}$ is also tested.

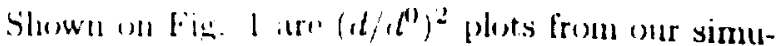
lations portraying . Vomura at al. $\therefore$ [20| experiments at high temperature $(i .15 k)$ in the pressure range of 0.1 - 2.MPa. In the unmerical simulations, the location of the drop boundary is defined to be that of the maximum density gratient, for consistency with optical measurements. In agreement with well known theory [12], at $0.1 \mathrm{MPa}$ the liquid/gas interface is found to be precisely that of maximum density gradient. With increasing $p$ the two locations still coincide for all simulations in the range $0.1-5 \mathrm{MPa}$ investigated in this work, but the density gradient, although still substantial, decreases across the boundary as $p$ increases.

All but two of our simulations were conducted with $\alpha_{I K}=0.01$; the remaining simulations was conducted with $\alpha_{B K}=0.01$. Our results with $\alpha_{I K}=0.01$ capture the $0.1 \mathrm{MPa}$ data very well but display a somewhat earlier $d^{2}$-law behavior; it is unclear whether the noncoinciding part of the data and simulations fall within the experimental error since this error is not provided with the data. However, the results for $0.1 \mathrm{MPa}$ with $\alpha_{B K}=0.01$ depart considerably from the data. Similarly, the data at $0.5 \mathrm{MPa}$ is compared with results from simulations with both $\alpha_{B K}$ and $\alpha_{I K}$ specified as 0.01 . It is clear that the $\alpha_{B K}=0.01$ results fall short of agreement with the data, and in fact show a typical large increase in the evaporation time that was obtained with $\alpha_{B K}=0.01$ at other pressures as well. In contrast, the $\alpha_{I K}=0.01$ results capture the nonlinear portion of the curve very well with a small discrepancy in the total evaporation time. Simulations and data at $2 \mathrm{MPa}$ agree only during the initial time, after which the simulations display the expected smooth variation consistent with drop heating, whereas the data exhibit two discontinuities that can be explained only by the presence of the suspending fiber. Calculated slopes of the linear part of the curves, called the evaporation constant [12], $K$, are presented for comparison in Table 1 for the 0.1 and $0.5 \mathrm{MPa}$ results obtained with $\alpha_{I K}=0.01$. Despite the presence of the suspending fiber in the experiments, an excellent agreement exists between simulations and data. A similar comparison cannot be performed at 2 $\mathrm{MPa}$ since there is no evidence of linear behavior in the data.

\section{Confirmation of thermal diffusion factors from} intermediate temperatures data

Displayed in Fig. 2 are $p=2 \mathrm{MPa}$ comparisons of simulation results at $655 \mathrm{~K}$ for various values of $\alpha_{I K}$, one simulation where $\alpha_{B K}$ instead of $\alpha_{I K}$ is prescribed, and Nomura et al.'s $20 \mid$ data at $656 \mathrm{~K}$. The numerical predictions are a very weak function of $\alpha_{I K}$ in the range $-0.6-0.6$ and agree remarkably well with the data during the initial heat up period of the drop. Eventually, the data shows a faster evaporation than our simulations, although the lack of error bars in the data make it impossible to evaluate the extent of the disagreement. 


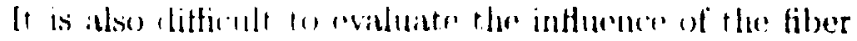
(during the experiment) on the sevaporition process. However, results with $k / \mathrm{k}=1) .01$ chesurly overestimate both the growth of the ctrop cluring the initial heat up time and the lrop evaporation time. These results are consistent with those of Fig. 1.

Additional comparisons between numerical predictions and data is portrayed in Fig. 3 where comparisons are made in the range $0.1-2 \mathrm{MPa}$ between simulations at $655 \mathrm{~K}$ with $\alpha_{I K}=0.01$, and data in the range 648 $669 \mathrm{~K}$. The initial heating time is again very well reproduced by the simulations, except that the predictions at $0.1 \mathrm{MPa}$ display again an earlier $d^{2}$-law behavior. The evaporation time is very well reproduced at $0.1 \mathrm{MPa}$, and less well as the pressure increases. Since it is difficult to quantify the influence of the suspending fiber on this data as the pressure increases, we can qualify this comparison as very encouraging.

Table 1 includes comparisons of $K$ for this intermediary temperature regime, and shows good to excellent agreement between data and predictions.

This study indicates (see also below) that the value of $\alpha_{I K} / \alpha^{*}$ is indeed rather small and that $\alpha_{B K} \simeq-\alpha_{h}$ is correct. The assumption made in all calculations presented below is that the value of $\alpha_{I K}$ is the same small value determined at high temperatures regardless of the $(p, T)$ conditions, and thus that $\alpha_{B K} \simeq-\alpha_{h}$.This assumption might not be entirely valid, as in general $\alpha_{I K}$ is a function of both $p$ and $T$. This assumption, the fact that the data is from suspended drop experiments whereas our calculations are for free drops, and the slight but nevertheless present influence of buoyancy for the stated microgravity level of $10^{-2}-10^{-3}$ in the experiment might explain the 15-20\% discrepancies (see below and Table 1) between data and results from simulations.

\section{Comparison with data at low temperatures}

The low temperature data of Nomura et al. $[20]$ and Sato [19] (Sato's data was approximated from his figure) is shown in Fig. 4 along with our numerical predictions at $445 \mathrm{~K}, 470 \mathrm{~K}$ and $495 \mathrm{~K}$ using $\alpha_{I K}=0.01$. The temperature range for Nomura et al.'s [20] data is 466 - $493 \mathrm{~K}$ whereas Sato's [19] data was obtained at 445 $K$; the data in $[20]$ is in the 0.1 - $5 \mathrm{MPa}$ range, whereas that of [19] is at $2 \mathrm{MPa}$. The comparisons are very good at low $p$ and deteriorate as $p$ increases. The predictions and data [20] agree remarkably well at 0.1 and $0.5 \mathrm{MPa}$, whereas at $1 \mathrm{MPa}$ the evaporation time is slightly overpredicted by the simulations. Nevertheless, the calculated and measured evaporation constant ( $\mathrm{Ta}$ ble 1) show very good agreement at all three pressures. The 2 MPa numerical results approximate the $d^{2}$ experimental variation [19] fairly well, and the agreement in the value of $K$ (Table 1 ) is excellent. At $p=5$ MPa, our simulation of a free drop shows an increased heating time, whereas the suspended drop in the exper-

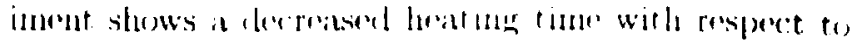
the 0.1 MPa case. [he litforener betwerll phe experimental conditions and those of the simulations explains the disagrement in the heat up time, although the rate of regression of the largest. gratient location is surprisingly well predicted. Since at is . $\mathrm{Pa}$ the conditions are supercritical, there is no evaporation and the concept of evaporation constant is irrelevant, although comparisons between the rates of regression are still meaningful.

\section{Determination of the Lewis number}

Figure 5 (a - d) illustrates the classical and the effective Lewis number, $L e$ and $L e_{e f f}$, for the high temperature calculations $(745 \mathrm{~K}$ ) validated above. Figures $5 \mathrm{a}$ and $5 \mathrm{~b}$ show results from the $0.1 \mathrm{MPa}$ simulations, whereas Figs. $5 \mathrm{c}$ and $5 \mathrm{~d}$ portray parallel results at $2 \mathrm{MPa}$; in both simulations the pressure is in the subcritical regime. Inspection of the plots shows that for a pure mixture at subcritical conditions $L e$ and $L e_{\text {eff }}$ have the same values in regions of weak gradients, thereby validating our model for $L e_{e f f}$. Quantitative and qualitative differences appear in mixture regions where gradients are important, and $L_{\text {eff }}$ can be as much as a factor of 2 larger than Le. With increasing pressure, while still in the subcritical regime, these differences become larger indicating that the estimate provided by Le regarding the relative importance of heat and mass diffusional scales deteriorates as the pressure increases.

Similar plots of $L e$ and $L e_{e f f}$ appear on Fig. 6 (a d) for the low temperature simulations validated above. Figures $6 \mathrm{a}$ and $6 \mathrm{~b}$ are for the same pressure as in Figs. $5 \mathrm{c}$ and $5 \mathrm{~d}$ ( $2 \mathrm{MPa}$ ), but at $445 \mathrm{~K}$, whereas Figs. $6 \mathrm{c}$ and $6 \mathrm{~d}$ are for $5 \mathrm{MPa}$ (supercritical pressure) and $495 \mathrm{~K}$. For the low temperature, subcritical pressure $L e$ and $L e_{\text {eff }}$ are in close agreement (Figs. $6 a$ and $6 \mathrm{~b}$ ), again validating our model for $L e_{e f f}$ and comparisons with Figs. $5 \mathrm{c}$ and $5 \mathrm{~d}$ shows that as expected, the lack of strong heating in Figs. $6 \mathrm{a}$ and $6 \mathrm{~b}$ decreases the gradients and increases the agreement of $L e$ and $L e_{e f f}$. However, even at low heating, under supercritical pressure conditions $L_{e f f}$ becomes substantially different from $L e$ as shown in Figs. $6 \mathrm{c}$ and $6 \mathrm{~d}$. Mistakenly, $L e$ indicates that the drop is gaseous $(L e<1)$, whereas $L e_{e} f f$ correctly indicates that the drop is a dense gas with properties approaching those of a liquid ( $L e_{e f f}>1$ ). This comparison emphasizes the importance of an appropriate model for the determination of the ratio of heat to mass diffusional scales which is both of academic and practical interest in that design engineers often determine combustions chamber dimensions based upon such estimates.

Fortuitously, the far field boundary conditions of the simulations (taken to be those of the data) presented in Figs 5 and 6 allowed the uncoupling of the pressure and temperature effects on the $L e$ versus Le ff. Wo further explored the combined effect of pres- 


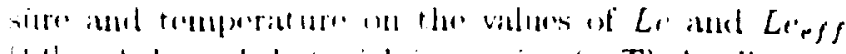
| 1 and showol that with incrobing (1, $T$ ) the discrepancy betweren the two estimates becomes substantially larger than shown here.

\section{Conclusions}

A model of fluid behavior under both sub- and supercritical thermodynamic conditions has been discussed with particular emphasis on the different physics according to the initial conditions with respect to the critical point. The model has been exercised for a fluid drop for which data are available for model validation. The drop is typically colder than its surroundings whose far field conditions are prescribed. In the subcritical regime and for large emission rates from the drop, there exists a film layer in the inner part of the drop surface and the solution of the equations has a convective-diffusive character. In the supercritical regime, there is no material surface to follow, and this introduces an indeterminacy in the boundary conditions. To resolve this indeterminacy one must follow an arbitrary boundary of interest which is here that of the initial lluid drop. The solution has then a pure diffusive character, and from this solution we calculate the location of the highest density gradient which we identify with the optically observable fluid drop.

The model was exercised for a heptane drop in nitrogen because of the existing data available for comparison. Simulations obtained with this model were validated with microgravity experimental data for large drops over a wide range of temperatures and pressures. The large temperature data were used to determine the value of the thermal diffusion factor and further validations were conducted with this fixed value. The agreement between predictions and $d^{2}$ data is excellent at atmospheric pressure and becomes fair at supercritical pressure, whereas the rate of regression of the point of maximum density gradient is remarkably well predicted at all pressures. The numerical predictions show that the traditional $d^{2}$-law is obeyed only in the subcritical regime. As the pressure is increased, $d^{2}$ becomes nonmonotonic with time, with a slope whose magnitude increases as a function of time. Thus, we initially identify a heating period during which the drop size may increase, followed by a period during which the size is continuously reduced. The duration of the heat-up period increases with far field pressure.

Analysis of the results shows that the classical Lewis number is not a good indicator of the ratio of heat to mass diffusion at high pressure or in the presence of strong gradients. Instead, an effectve Lewis number previously defined is shown to give a qualitatively consistent indication of the relative scales of heat and mass diffusion.

\section{ACKNOWLEDGMENT}

This research was conducted at the Jet Propulsion Laboratory under sponsorship from the National Aero-

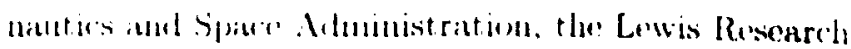
Cenuter with Dr. Daniel L. Bulzan as terdenical contract monitor. His continuing interest and support are greatly appreciated. We also thank Prof. Hiroshi No mura of Nihou Univrrsity in Chiba, Japan, and Prof. Iskender Goskalp of the Centre National de la Recherche Scientifique in Orléans. France, for providing us with their data.

\section{References}

(1) Mayer, W., Labani. R. and Krulle, G., "Theoretical investigations of droplet flow under typical coaxial injector flow conditions in cryogenic rocket engines", AIAA 92-3121, $28^{\text {th }}$ Joint Propulsion Conference, Nashville, TN, 1992

[2] Krulle, G. and Mayer, W., "Injection, atomization and mixing of propellants in liquid rocket engines using coaxial injectors", Proc. ICLASS-94, Rouen, France, 1994

[3] Harstad, K. and Bellan, J., "Isolated fluid oxygen drop behavior in fluid hydrogen at rocket chamber pressures", Int. J. Heat Mass Transfer, 1998a, 41, $3537-3550$

[4] Mayer, W., Schik, A., Schweitzer, C. and Schaffler, M., "Injection and mixing processes in high pressure LOX/GH2 rocket combustors", AIAA 962620

[5] Mayer, W., Ivancic, B., Schik, A. and Hornung, U., "Propellant atomization in LOX/GH2 rocket combustors", AIAA 98-3685

[6] Chehroudi, B., Talley and Coy, E., "Initial growth rate and visual characteristics of a round jet into a sub- to supercritical environment of relevance to rocket, gas turbine and Diesel engines", AIAA 99 0206

[7] Harstad, K. and Bellan, J., "The Lewis number under supercritical conditions", Int. J. Heat Mass Transfer, 42, 961-970, 1999

[8] Yang, V., Lin, N. and Shuen, J-S., "Vaporization of liquid oxygen (LOX) droplets in supercritical hydrogen environments", Combust. Sci. and Tech., 1994, 97, 247-270

[9] Delplanque, J-P. and Sirignano, W. A., "Numerical study of the transient vaporization of an oxygen droplet at sub- and super-critical conditions". Int. J. Heat Mass Transfer, 1993, 36(2), 303-314

(10) Haldenwang, P., Nicoli, C. and Daou, J., "High pressure vaporization of LOX droplet crossing the critical condition", Int. J. Heat Mass Transfer. 1996, 39(16), 3453-3464 
(11) Givler, S. D. and Aluraham, J., "Supercriti(al ilroplet vnporization and rombustion studies", Proy. Entergy Combust. Sci., 1996, 22, 1-28

[12) Williams, F. A., Combustumn Theory, AddisonWesley, 1965

[13] Keizer, J., Statistical Thermodynamics of Nonequilibrium Processes, Springler-Verlag, New York, 1987

(14) Harstad, K. and Bellan, J., "An all-pressure fluiddrop model applied to a binary mixture: heptane in nitrogen", submitted for publication

[15] Law, C. K. and Law, H. K., "A d"law for multicomponent droplet vaporization and combustion", AIA A Journal, 20, 4, 522-527, 1982

[16] Harstad, K. G. , Miller, R. S., and Bellan, J., "Effcient high pressure state equations", $A . I$. Ch. $E$., $43(6), 1605-1610,1997$

[17] Vieille, B., Chauveau, C., Chesnau, X, Odeide, A. and Gokalp, I., "High-pressure droplet burning experiments in microgravity", $26^{\text {th }}$ Symp. (Int). on Combustion, 1259-1265, 1996

[18] Morin, C., "Studies on the influence of pressure and temperature on the vaporization of hydrocarbon droplets", Ph.D. thesis, Centre National de la Recherche Scientifique, Orleans, France, 1999

[19] Sato, J., "Studies on droplet evaporation and combustion in high pressures", AIAA 93-0813, $31^{\text {st }}$ Aerospace Sciences Meeting, Jan. 11-14, Reno, NV

[20] Nomura, H., Ujiie, Y., Rath, H. J., Sato, J. and Kono, M., "Experimental study on high pressure droplet evaporation using microgravity conditions", 26 $6^{\text {th }}$ Symp. (Int.) on Comb., 1996, 1267-1273

[21] Chauveau, C., Chesnau, X. and Gokalp, I., "Burning characteristics of n-heptane droplets", AIAA 93-0824, 31st Aerospace Sciences Meeting, Reno, NV., 1993

[22] Morin, C., Chauveau, C. and Gokalp, I., "Studies on the influence of pressure and temperature on the vaporization of n-heptane droplets", personal communication, to be presented at the ILASS-Europe 1999 meeting

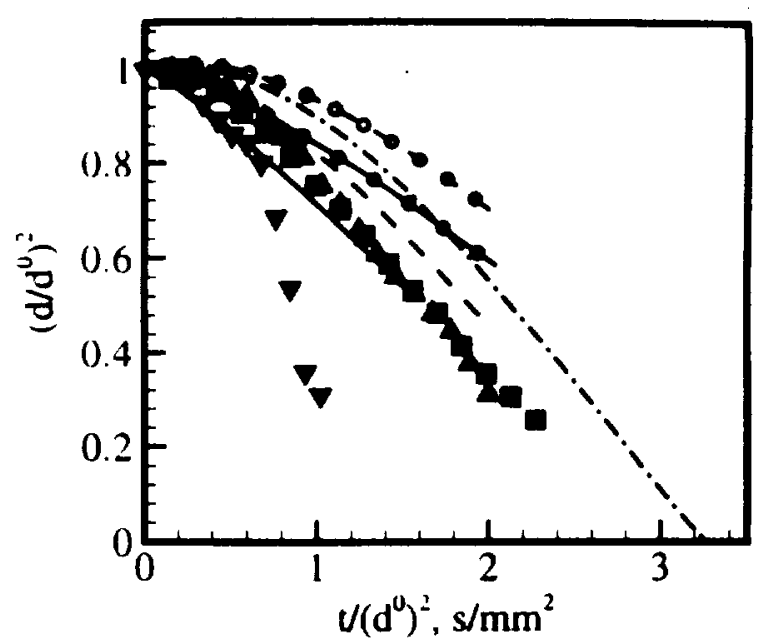

Fig. 1 High temperature comparisons. $R_{d}^{0}=0.35$ $\mathrm{mm} ; R_{e}^{0}=4 \mathrm{~mm}, Y_{e}^{0}=0$ and $T_{d, b}^{0}=300 \mathrm{~K}$. In the far field $T_{e}$ and $p_{e}$ are specified as in the experiments. Simulations at $T_{e}=745 \mathrm{~K}$ and $p_{e}: 0.1 \mathrm{MPa}, \alpha_{I K}=$ $0.01-; 0.1 \mathrm{MPa}, \alpha_{B K}=0.01-; 0.5 \mathrm{MPa}, \alpha_{I K}=$ 0.01 - - ; $0.5 \mathrm{MPa}, \alpha_{B K}=0.01 \ldots-$ - $2 \mathrm{MPa}-\cdots$. Data: $741 \mathrm{~K}$ and $0.1 \mathrm{MPa} \square ; 749 \mathrm{~K}$ and $0.5 \mathrm{MPa} \triangle$; $746 \mathrm{~K}$ and $2 \mathrm{MPa} \nabla$.

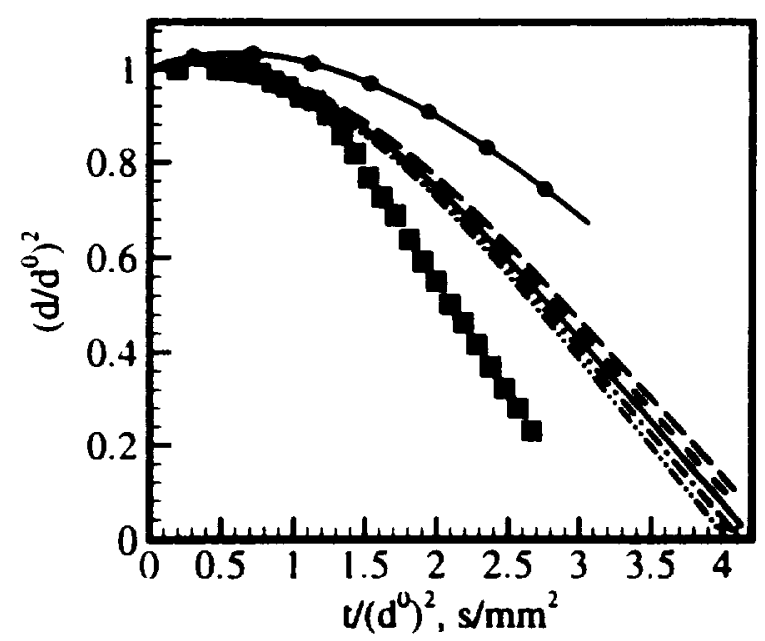

Fig. 2 Intermediary temperature comparisons at $2 \mathrm{MPa} . \quad R_{d}^{0}=0.35 \mathrm{~mm} ; R_{e}^{0}=4 \mathrm{~mm}, Y_{e}^{0}=0$ and $T_{d, b}^{0}=300 \mathrm{~K}$. Simulations at $655 \mathrm{~K} ; \alpha_{I K}=0.01-$ 0.3 - - ; - $0.3-\cdots ;-0.6-\cdots-; 0.6--; \alpha_{B K}=0.01$ - - Data at $656 \mathrm{~K}$ : 


\begin{tabular}{|c|c|c|c|c|c|}
\hline$p_{1} \ldots M l_{u}$ & $T, K^{\circ}$ & $\pi(\mu t y)$ & $k$ (normat & $A_{.1,}$ & 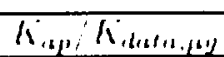 \\
\hline 0.1 & .170 & $0.116(\mathrm{Nom})$ & 0.22 (Nor) & 0.135 & 1.16 \\
\hline 0.1 & 6555 & $0.306(\mathrm{Nom})$ & 0.45 (Mor) & 0.280 & 11.92 \\
\hline 0.1 & 745 & $0.390(\mathrm{Nom})$ & $0.56(\mathrm{M} o r)$ & 0.3 .50 & $(1.90$ \\
\hline 0.7 & 470 & 0.117 (Nom) & & 0.135 & 1.15 \\
\hline 0.5 & 655 & 0.356 (Nom) & & 0.320 & 0.90 \\
\hline 0.5 & 745 & 0.437 (Nom) & & 0.390 & 0.89 \\
\hline 1.0 & 470 & 0.138 (Nom) & & 0.135 & 0.98 \\
\hline 1.0 & 655 & 0.424 (Nom) & & 0.330 & 0.78 \\
\hline 2.0 & 445 & 0.097 (Sat) & 0.14 (Sat) & 0.0935 & 0.97 \\
\hline 2.0 & 450 & 0.096 (Nom) & & & \\
\hline 2.0 & 655 & 0.475 (Nom) & & 0.360 & 0.76 \\
\hline 2.0 & 745 & $0.4-1.7$ (Nom) & & 0.450 & - \\
\hline 5.0 & 495 & $0.2-0.13$ (Nom) & & 0.140 & $0.70-1.08$ \\
\hline
\end{tabular}

Table 1: Maximum regression rate of the maximum density gradient location, $K$ in $\mathrm{mm}^{2} / \mathrm{s}$, obtained from the current model (ap), Nomura et al.'s microgravity experimental data (Nom), Sato's microgravity and normal gravity experimental data (Sat), and Morin et al.'s normal-gravity data (Mor). The Nomura et al.'s and Morin et al.'s data were provided by the authors, and Sato's values were read on their graph following the directions given in their paper. In the simulations $T_{d}^{0}=300 \mathrm{~K}$ and $d^{0}=0.7 \mathrm{~mm}$, while Nomura et al.'s $d^{0}$ was $0.6-0.8 \mathrm{~mm}$, Sato's was $1 \mathrm{~mm}$, and Morin et al.'s was $1-1.5 \mathrm{~mm}$.

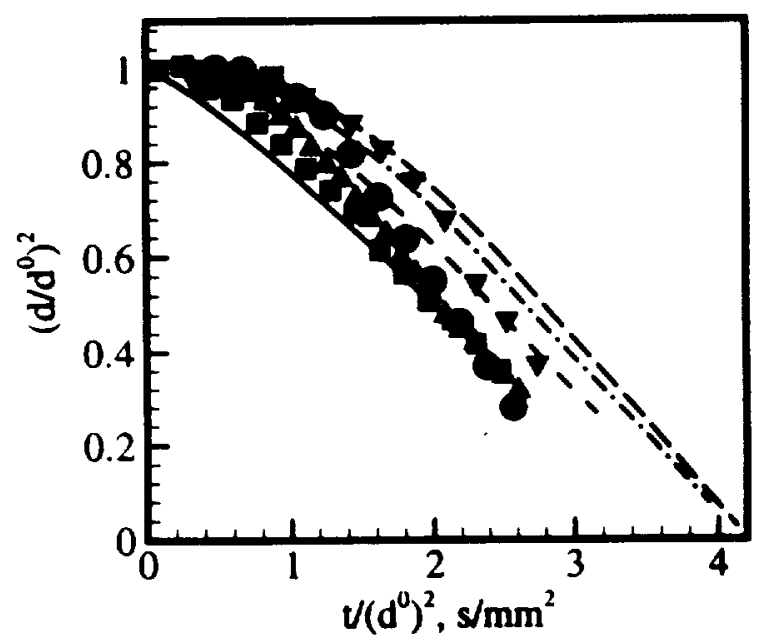

Fig. 3 Intermediary temperature comparisons. $R_{d}^{0}=0.35 \mathrm{~mm} ; R_{e}^{0}=4 \mathrm{~mm}, Y_{e}^{0}=0$ and $T_{d, b}^{0}=300$ $\mathrm{K}$. Simulations at $655 \mathrm{~K}: 0.1 \mathrm{MPa}-; 0.5 \mathrm{MPa}-\cdots$; $1 \mathrm{MPB}-\cdot-; 2 \mathrm{MPa}$ - - Data: $648 \mathrm{~K}$ and $0.1 \mathrm{MPa}$; $655 \mathrm{~K}$ and $0.5 \mathrm{MPa} \triangle ; 669 \mathrm{~K}$ and $1 \mathrm{MPa \nabla} ; 656 \mathrm{~K}$ and $2 \mathrm{MPa} \bullet$.

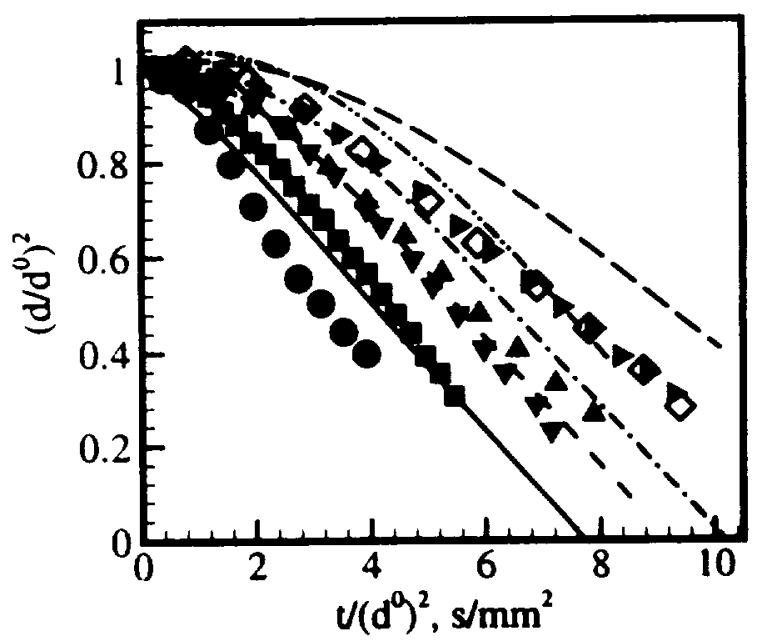

Fig. 4 Low temperature comparisons. $R_{d}^{0}=0.35$ $\mathrm{mm}$ except at $445 \mathrm{~K}$ where $R_{d}^{0}=0.5 \mathrm{~mm} ; R_{e}^{0}=4 \mathrm{~mm}$, $Y_{e}^{0}=0$ and $T_{d, b}^{0}=300 \mathrm{~K}$. Simulations at $470 \mathrm{~K}: 0.1$ $\mathrm{MPa}-; 0.5 \mathrm{MPa}-\cdot-; 1 \mathrm{MPa}-\cdot-;$ at $445 \mathrm{~K}$ and $2 \mathrm{MPa}-$ - ; at $495 \mathrm{~K}$ and $5 \mathrm{MPa}-\cdots$. Data: 471 $\mathrm{K}$ and $0.1 \mathrm{MPa} \square ; 468 \mathrm{~K}$ and $0.5 \mathrm{MPa} \triangle ; 466 \mathrm{~K}$ and 1 $\mathrm{MPa} \nabla ; 445 \mathrm{~K}$ and $2 \mathrm{MPa} \diamond ; 452 \mathrm{~K}$ and $2 \mathrm{MPa} \triangleright ; 493$ $\mathrm{K}$ and $5 \mathrm{MPa} \cdot$ 


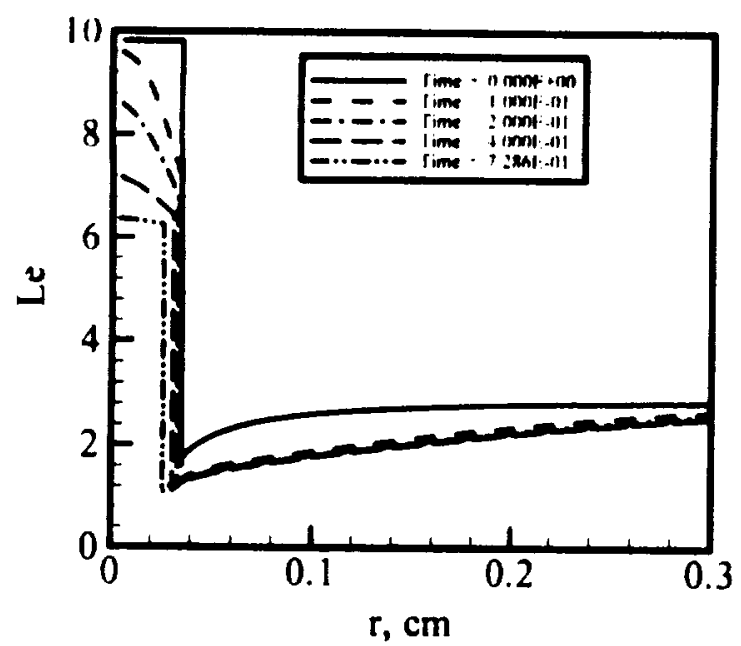

$\mathbf{2}$

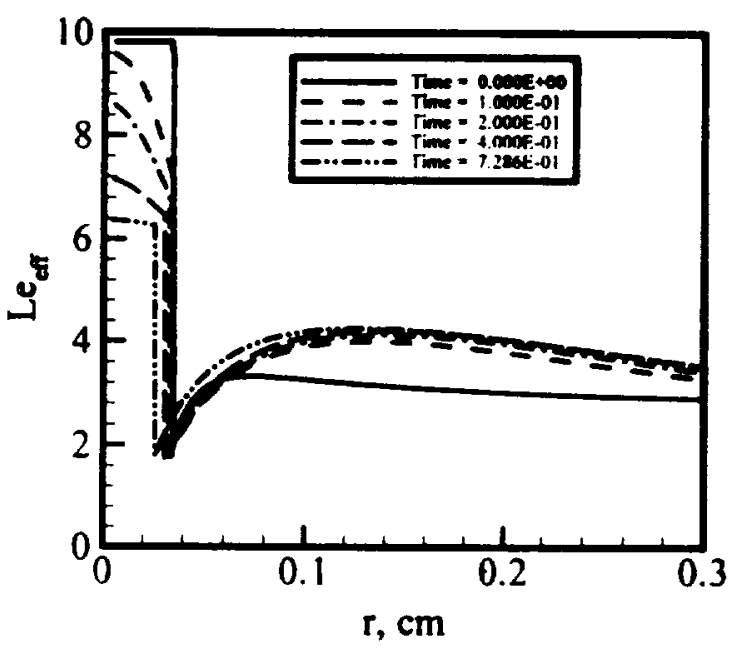

b

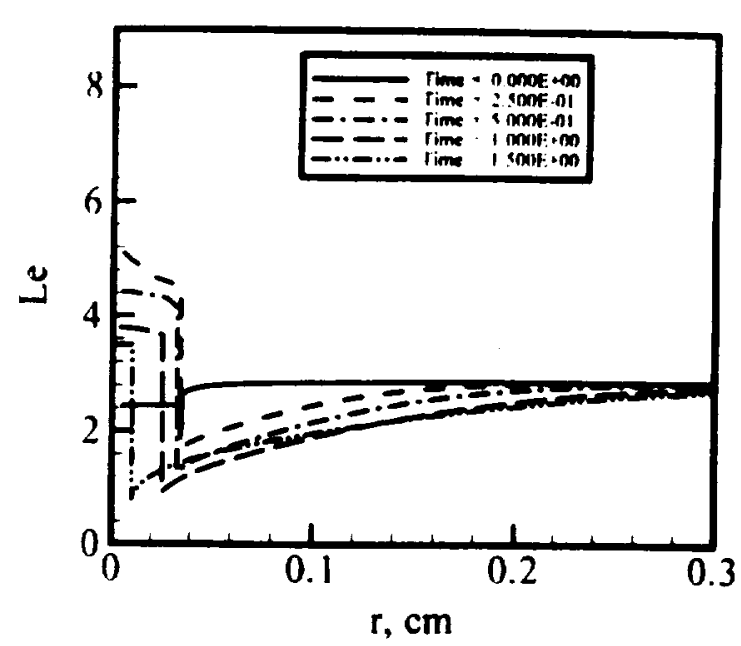

c

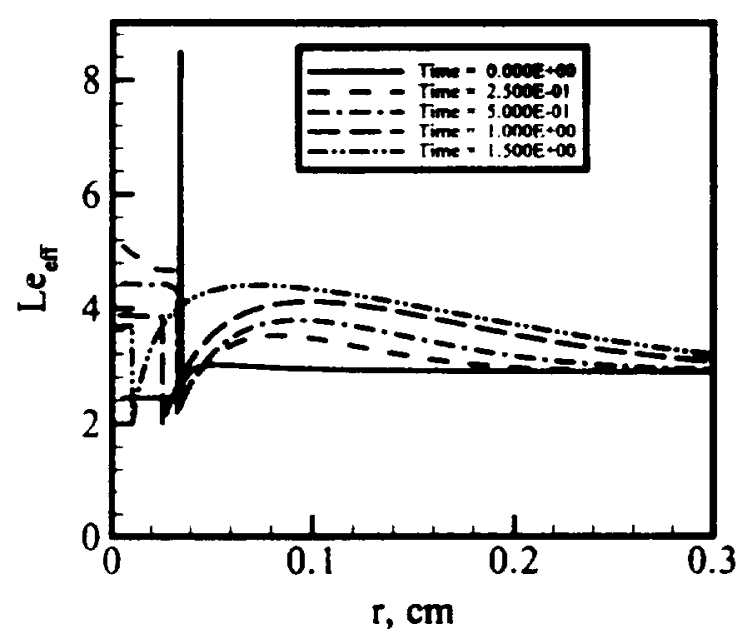

d

Fig. 5 Classical Lewis number, $L e$, and effective Lewis number, $L e_{e / f}$, at $T_{a}=745 \mathrm{~K}$ versus radial location at different times specified in $s$ in the legend. $p_{e}=$ $0.1 \mathrm{MPa}$ in (a) and (b); $2 \mathrm{MPa}$ in (c) and (d). 


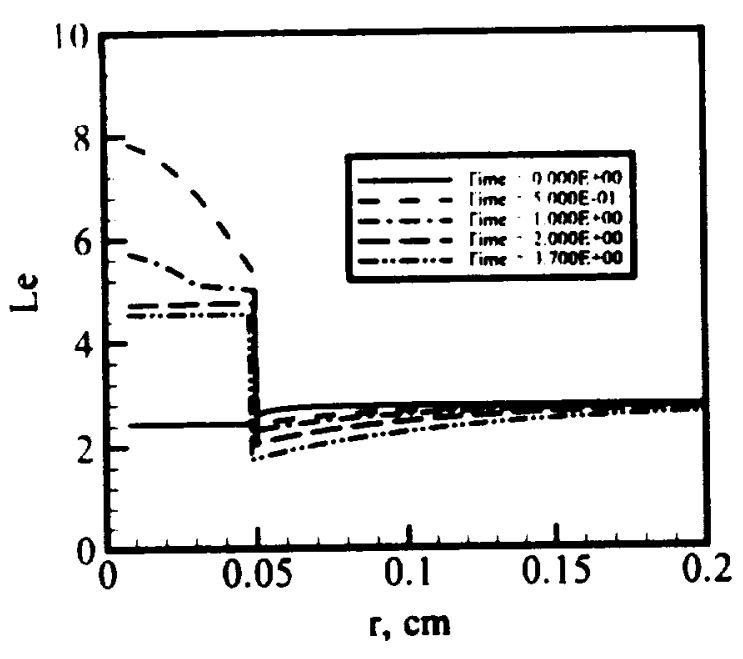

a

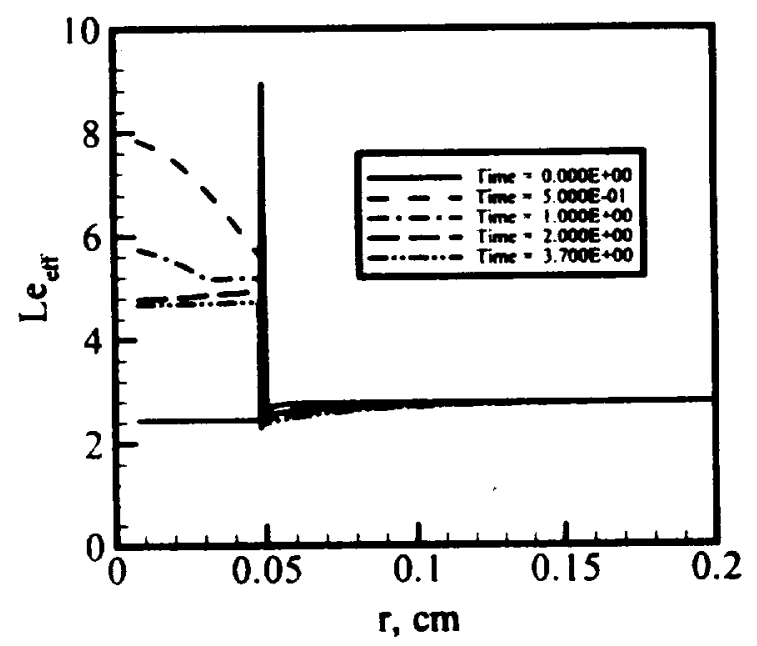

b

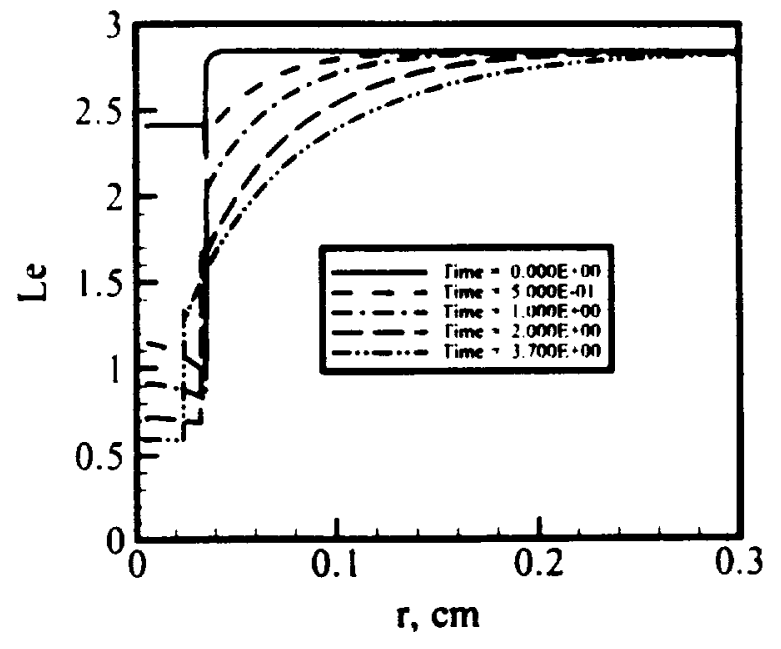

C

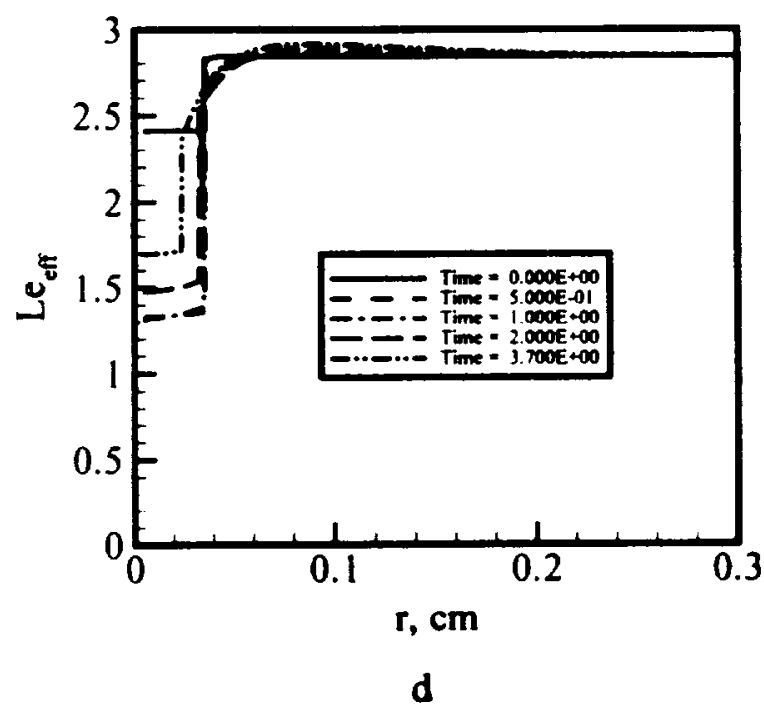

Fig. 6 Classical Lewis number, Le, and effective Lewis number, $L e_{e f f}$, versus radial location at different times specified in $s$ in the legend. (a) and (b) $p_{e}=2$ $\mathrm{MPa}$ and $T_{e}=445 \mathrm{~K} ;(\mathrm{c})$ and (d) $p_{e}=5 \mathrm{MPa}$ and $T_{e}=$ $495 \mathrm{~K}$. 